\title{
Discrete-Time Immersion and Invariance Adaptive Control of a Slider-crank Mechanism
}

\author{
Yaprak Yalçın* \\ *Dept. of Control Engineering, Istanbul Technical University, \\ Maslak, 34469, Istanbul, Turkey; yalciny@itu.edu.tr.
}

\begin{abstract}
A discrete-time adaptive nonlinear control procedure is developed based on immersion and invariance control, and using back-stepping for the regulation of slider-crank system. A convenient parametric formulation of the system dynamics is established. A novel parameter estimator design is presented, and the back-stepping controller is constructed considering the certainty equivalence principle.
\end{abstract}

\section{INTRODUCTION}

The slider-crank mechanism has a very wide usage in machine design. Some of the applications are found in internal combustion engines, in electrical switch gears, packaging and textile engineering (Lin et al. 1997, Ha et al. 2006). It is mainly used to convert rotary motion to a reciprocating motion or vice versa. Several control techniques have been presented for the system in the literature by sliding mode control (Fung and Chang, 2009), energy control (Komaita and Furuta, 2008), generalized minimum variance control (Saitoh and Furuta, 2007) and computed torque technique (Lin et al. 1998).

Most of the mechanisms in the engineering applications consist of motors, gears and nonlinear components. The control performance of these systems is usually dominated by the effects of nonlinearities and uncertainties. Therefore, the nonlinear adaptive design procedure for the case study considered in this paper may be applicable for a wider range of nonlinear electro-mechanical systems.

On the other hand, to develop modelling and control techniques for discrete-time nonlinear systems have gained more importance as in the engineering practice computercontrolled systems are preferred because of the simplicity and flexibility of their implementation.

Immersion and Invariance (I\&I) control is a recently presented new methodology in the literature proposed by (Astolfi et al., 2007, Astolfi et al., 2008, Karagiannis and Astolfi 2008) for designing nonlinear and adaptive controllers for nonlinear systems which relies upon the notion of system immersion and manifold invariance. In I\&I control approach the behaviour that satisfies the control objective is formulated as a target system and the controller is constructed such that the plant dynamics immersed into the target dynamics.

In (Yalcin and Astolfi 2011, 2012) a discrete-time adaptive controller design via state-feedback for the adaptive regulation of linearly parameterised discrete-time systems in strict-feedback form is presented. In this paper, the adaptive regulation of the slider-crank mechanism is considered and a discrete time adaptive nonlinear control procedure is proposed based on immersion and invariance control approach and using back-stepping. For the purpose, firstly, a parametric model for the system dynamics is obtained. The system dynamics are not directly linear in parameters but it is expressed in a form such that the intended control procedure can be developed utilizing it. Then, a parameter estimator is designed. Afterwards the back-stepping controller is constructed considering certainty equivalence principle.

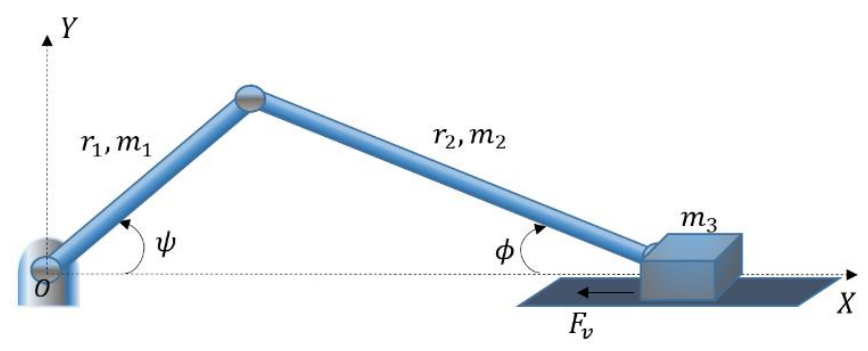

Fig. 1. Slider-crank systems

\section{DERIVATION OF SYSTEM DYNAMIC EQUATIONS}

In this section the dynamic equations for the considered crank-slide mechanism shown in Figure 1 are derived by the Euler-Lagrange technique and these equations are expressed in a form in the next section such that an immersion and invariance adaptive control algorithm can be developed and a back-stepping controller can be constructed.

In Figure 1, $r_{1}$ and $r_{2}$ are length of the arms, $m_{1}, m_{2}$ and $m_{3}$ masses of the arms and the slider, respectively. The slidercrank system is a 2-dof under-actuated mechanical system. The actuation is performed through the base joint. The angular motion of base joint is transformed to a linear motion in the slider so that the slider moves accordingly.

Let's write the kinetic energy function of the system,

$$
K=\frac{1}{2} I_{1} \dot{\psi}^{2}+\frac{1}{2} I_{2} \dot{\phi}^{2}+\frac{1}{2} m_{2} \vartheta_{2}^{2}+\frac{1}{2} m_{3} \vartheta_{3}^{2}
$$


where $I_{1}$ and $I_{2}$ are the inertias of arms, $\vartheta_{2}$ is the linear velocity of the second arm and $\vartheta_{3}$ is linear velocity of the mass $m_{3}$. Besides, consider the potential energy of the system as

$P=m_{1} g \frac{r_{1}}{2} \sin \psi+m_{2} g \frac{r_{2}}{2} \sin \phi$.

Note that the following relation is valid for the angles $\psi, \phi$,

$r_{1} \sin \psi=r_{2} \sin \phi$

Therefore,

$r_{1} \dot{\psi} \cos \psi=r_{2} \dot{\phi} \cos \phi$, namely $\dot{\phi}=\frac{r_{1} \dot{\psi} \cos \psi}{r_{2} \cos \phi}$.

Now consider $x_{c 2}=r_{1} \cos \psi+\frac{r_{2}}{2} \cos \phi$ and $y_{c 2}=\frac{r_{2}}{2} \sin \phi$ together with (3), then

$\vec{\vartheta}_{2}=\vartheta_{2 x} i+\vartheta_{2 y} j$

where

$\vartheta_{2 x}=\dot{x}_{c 2}=-r_{1} \dot{\psi} \sin \psi-\frac{r_{2}}{2} \dot{\phi} \sin \phi=-r_{1} \dot{\psi} \sin \psi-\frac{r_{1}}{2} \dot{\phi} \sin \psi$,

$\vartheta_{2 y}=\dot{y}_{c 2}=\frac{r_{2}}{2} \dot{\phi} \cos \phi=\frac{r_{1}}{2} \dot{\psi} \cos \psi$

therefore,

$\vartheta_{2}^{2}=r_{1}^{2} \sin ^{2} \psi\left(\dot{\psi}+\frac{1}{2} \dot{\phi}\right)^{2}+\frac{r_{2}^{2}}{4} \dot{\phi}^{2} \cos ^{2} \phi$

Here note that, using $\cos ^{2} \phi=1-\sin ^{2} \phi$ and relations (3-4), the following expression for $\dot{\phi}$ which is utilized in the sequel is obtained.

$\dot{\phi}=\frac{r_{1} \dot{\psi} \cos \psi}{\sqrt{r_{2}^{2}-r_{1}^{2} \sin ^{2} \psi}}$ equivalently $\dot{\phi}^{2}=\frac{r_{1}^{2} \dot{\psi}^{2} \cos ^{2} \psi}{r_{2}^{2}-r_{1}^{2} \sin ^{2} \psi}$.

Thus $\vartheta_{2}^{2}$ can be written as,

$$
\begin{aligned}
\vartheta_{2}^{2} & =r_{1}^{2} \sin ^{2} \psi\left(\dot{\psi}+\frac{1}{2} \dot{\phi}\right)^{2}+\frac{r_{2}^{2}}{4} \dot{\phi}^{2} \cos ^{2} \phi \\
& =r_{1}^{2} \sin ^{2} \psi \dot{\psi}^{2}+\frac{r_{1}^{3} \dot{\psi}^{2}}{c} \sin ^{2} \psi \cos \psi+\frac{r_{1}^{2} r_{2}^{2} \dot{\psi}^{2}}{4 c^{2}} \cos ^{2} \psi \\
& =r_{1}^{2} \dot{\psi}^{2}\left[\sin ^{2} \psi\left(1+\frac{r_{1}}{c} \cos \psi\right)+\frac{r_{2}^{2}}{4 c^{2}} \cos ^{2} \psi\right]
\end{aligned}
$$

where

$c=\sqrt{r_{2}^{2}-r_{1}^{2} \sin ^{2} \psi}$

as in (Ha et al. 2006).

Finally, since $x_{c 3}=x=r_{1} \cos \psi+r_{2} \cos \phi$ and $y_{c 3}=0$, then

$$
\begin{aligned}
\vartheta_{3}=\dot{x} & =-r_{1} \dot{\psi} \sin \psi-r_{2} \dot{\phi} \sin \phi \\
& =-r_{1} \dot{\psi} \sin \psi-r_{1} \dot{\phi} \sin \psi \\
& =-r_{1} \dot{\psi} \sin \psi\left(1+\frac{r_{1}}{c} \cos \psi\right)
\end{aligned}
$$

and when (9) is considered,

$$
\begin{aligned}
\vartheta_{3}^{2} & =\dot{x}=r_{1}^{2} \sin ^{2} \psi(\dot{\psi}+\dot{\phi})^{2} \\
& =r_{1}^{2} \dot{\psi}^{2} \sin ^{2} \psi+\frac{2 r_{1}^{3} \dot{\psi}^{2}}{c} \sin ^{2} \psi \cos \psi \\
& +\frac{r_{1}^{4} \dot{\psi}^{2}}{c^{2}} \sin ^{2} \psi \cos ^{2} \psi .
\end{aligned}
$$

Moreover, the potential energy of the system can be rewritten as,

$$
P=m_{1} g \frac{r_{1}}{2} \sin \psi+m_{2} g \frac{r_{2}}{2} \sin \phi=\left(m_{1}+m_{2}\right) g \frac{r_{1}}{2} \sin \psi .
$$

Thus, the Lagrangian for the system is obtained as follows,

$$
\begin{aligned}
L=K-P & =\frac{1}{2} I_{1} \dot{\psi}^{2}+\frac{1}{2} I_{2} \frac{r_{1}^{2} \dot{\psi}^{2} \cos ^{2} \psi}{c^{2}} \\
& +\frac{1}{2} m_{2} r_{1}^{2} \dot{\psi}^{2}\left[\sin ^{2} \psi\left(1+\frac{r_{1}}{c} \cos \psi\right)+\frac{r_{2}^{2}}{4 c^{2}} \cos ^{2} \psi\right] \\
& -\frac{1}{2} m_{3} r_{1} \dot{\psi} \sin \psi\left(1+\frac{r_{1}}{c} \cos \psi\right)-\left(m_{1}+m_{2}\right) g \frac{r_{1}}{2} \sin \psi .
\end{aligned}
$$

Hence, considering the inertias as $I_{1}=\frac{1}{\lambda_{1}} m_{1} r_{1}^{2}$ and $I_{2}=\frac{1}{\lambda_{2}} m_{2} r_{2}^{2}$ to capture the various arm-joint structures and uncertainties, and $F_{v}=-\operatorname{sgn}(\dot{x}) f_{v} \dot{x}=-\operatorname{sgn}(\dot{x}) \mu_{v} m_{3} g \dot{x} \quad$ the Lagrange Equations of the system is obtained as given below,

$\frac{d}{d t}\left(\frac{\partial L}{\partial \dot{\psi}}\right)-\frac{\partial L}{\partial \psi}=\left(k_{1}+P_{1}(\psi) \cos ^{2} \psi+P_{2}(\psi) \sin ^{2} \psi\right) \ddot{\psi}$

$+\left[\left(2 k_{3} \sin \psi \cos ^{2} \psi \dot{\psi}-2 P_{1}(\psi) \dot{\psi}+2 P_{2}(\psi)\right) \sin \psi \cos \psi\right.$

$\left.-k_{5} \dot{\psi} \sin ^{3} \psi-\dot{\psi} \sin \psi P_{3}(\psi)\right] \dot{\psi}+k_{10} \cos \psi=\tau-F_{v}$

Here, $\tau$ is the torque applied to base joint, $F_{v}$ is the viscous friction, $\mu_{v}$ is the viscous friction coefficient, $\lambda_{1}, \lambda_{2}$ are positive scalars and

$$
\begin{aligned}
& \begin{aligned}
P_{1}(\psi) & =k_{2}+k_{3} \sin ^{2} \psi, \\
P_{2}(\psi) & =k_{4}+k_{5} \cos \psi, \\
P_{3}(\psi)= & k_{6} \cos \psi\left(c+r_{1}^{2} \cos ^{2} \psi\right)+k_{4} \cos \psi+k_{7}\left(2 \cos ^{2} \psi\right. \\
& \left.\quad-\sin ^{2} \psi\right)-k_{8} \cos \psi+k_{9} \cos \psi\left(\cos ^{2} \psi-\sin ^{2} \psi\right),
\end{aligned} \\
& F_{v}=-\operatorname{sgn}(\dot{x}) f_{v} \dot{x}=-k_{11} \operatorname{sgn}(\dot{x}) \sin \psi\left(1+\frac{r_{1}}{c} \cos \psi\right) \dot{\psi}, \\
& \text { with }
\end{aligned}
$$




$$
\begin{aligned}
& k_{1}=\frac{m_{1} r_{1}^{2}}{\lambda_{1}}, k_{2}=\frac{5 m_{2} r_{2}^{2} r_{1}^{2}}{4 c^{2}}, k_{3}=\frac{r_{1}^{4} m_{3}}{c^{2}}, k_{4}=r_{1}^{2}\left(m_{2}+m_{3}\right), \\
& k_{5}=\frac{r_{1}^{3}\left(m_{2}+2 m_{3}\right)}{c}, k_{6}=-\frac{r_{1}^{2} r_{2}^{2} m_{2}}{c^{4} \lambda_{2}}, k_{7}=\frac{r_{1}^{3}}{2 c}\left(m_{2}+m_{3}\right), \quad(17) \\
& k_{8}=\frac{m_{2} r_{1}^{2} r_{2}^{2}}{4 c^{2}}, k_{9}=\frac{m_{3} r_{1}^{4}}{2 c}, k_{10}=\left(m_{1}+m_{2}\right) g \frac{r_{1}}{2}, \\
& k_{11}=f_{v} r_{1}=\mu_{v} m_{3} g r_{1} .
\end{aligned}
$$

Note that, the equations (15)-(17) can be written in the form of

$$
A(\psi) \ddot{\psi}+B(\psi, \dot{\psi}) \dot{\psi}+C(\psi)=\tau
$$

where

$A(\psi)=k_{1}+P_{1}(\psi) \cos ^{2} \psi+P_{2}(\psi) \sin ^{2} \psi$,

$B(\psi, \dot{\psi})=\left(2 k_{3} \sin \psi \cos ^{2} \psi \dot{\psi}-2 P_{1}(\psi) \dot{\psi}+2 P_{2}(\psi)\right) \sin \psi \cos \psi$ $-k_{5} \dot{\psi} \sin ^{3} \psi-\dot{\psi} \sin \psi P_{3}(\psi)+k_{11} \operatorname{sgn}(\dot{x}) \sin \psi \times\left(1+\frac{r_{1}}{c} \cos \psi\right)$, $C(\psi)=k_{10} \cos \psi$.

Finally, considering (18) for $x_{1}=\psi, x_{2}=\dot{\psi}$, the dynamic equations of the system can be written as,

$$
\begin{aligned}
& \dot{x}_{1}=x_{2} \\
& \dot{x}_{2}=-\frac{B\left(x_{1}, x_{2}\right)}{A\left(x_{1}\right)} x_{2}-\frac{C\left(x_{1}\right)}{x_{1} A\left(x_{1}\right)} x_{1}+v .
\end{aligned}
$$

where $\tau=A\left(x_{1}\right) v$

\section{CONTROLLER DESIGN}

In this section, a discrete-time adaptive nonlinear control procedure is developed. The nonlinear system (23) can be considered as a time varying linear system. If all the parameters are known, an adaptive linear controller can be designed. In this case $A(\theta), \quad B(\theta, \dot{\theta})$ and $C(\theta)$ can be calculated at each sample and the linear controller to be designed can be accordingly adapted. If there is uncertainties in the parameters of the system, a nonlinear adaptive controller is needed or preferred to design, since it may not be possible to directly calculate the values of $\mathrm{A}, \mathrm{B}$ and $\mathrm{C}$ polynomials.

Here, it is assumed that $r_{1}$ and $r_{2}$ are known and $m_{1}, m_{2}, m_{3}$, $I_{1}$ and $I_{2}$ (namely $\lambda_{1}$ and $\lambda_{2}$ ) are unknowns and a discrete-time adaptive nonlinear controller based on immersion and invariance control approach is designed.

Therefore, let's rewrite system dynamics (19) in the following form,

$$
\begin{aligned}
& \dot{x}_{1}=x_{2} \\
& \dot{x}_{2}=\frac{\hat{\Phi}_{2 B C}\left(x_{1}, x_{2}\right) \theta}{\hat{\Phi}_{2 A}\left(x_{1}, x_{2}\right) \theta}+v,
\end{aligned}
$$

$\theta=\left[\begin{array}{lllll}\hat{k}_{1} & \hat{k}_{2} & \hat{k}_{3} & \hat{k}_{4} & \hat{k}_{5}\end{array}\right]^{T}$

where

$$
\begin{array}{r}
\hat{\Phi}_{2 A}\left(x_{1}\right)=\left[r_{1}^{2}, \frac{5 r_{2}^{2} r_{1}^{2}}{4 c^{2}} \cos ^{2} x_{1}+r_{1}^{2} \sin ^{2} x_{1}\left(1+\frac{r_{1}}{c} \cos x_{1}\right),\right. \\
\left.r_{1}^{2} \sin ^{2} x_{1}\left(\frac{r_{1}^{2}}{c^{2}} \cos ^{2} x_{1}+1\right)+\frac{2 r_{1}^{3}}{c} \cos x_{1} \sin ^{2} x_{1}, 0,0\right]
\end{array}
$$

and,

$$
\begin{gathered}
\hat{\Phi}_{2 B C}\left(x_{1}, x_{2}\right)=\left[0, \frac{r_{1}^{2} x_{2}}{2 c^{2}} \sin x_{1} \cos x_{1}\left(-5 r_{2}^{2} x_{2}+2 c^{2}\left(2-x_{2}\right)\right.\right. \\
\left.+2 c r_{1}\left(1-x_{2}\right) \cos x_{1}+\frac{c^{2} r_{2}^{2} x_{2}^{2}}{2}\right)+\frac{r_{1}^{3} x_{2}^{2}}{2 c} \sin ^{3} x_{1} \\
r_{1} \cos x_{1} \sin x_{1}\left(\frac{2 r_{1}^{3} x_{2}^{2}}{c^{2}} \sin x_{1} \cos ^{2} x_{1}+r_{1} x_{2}\left(2-x_{2}\right)\right. \\
-2 x_{2}^{2} \sin ^{2} x_{1}+\frac{2 r_{1}^{2}}{c} x_{2} \cos x_{1}-\frac{r_{1}^{3} x_{2}^{2}}{c}\left(\sin ^{2} x_{1}\right. \\
\left.\left.-\cos ^{2} x_{1}\right)+\left(2-x_{2}\right) x_{2} \cos x_{1}\right)+\frac{3 r_{1}^{3} x_{2}^{2}}{2 c} \sin ^{3} x_{1} \\
+\mu_{v} g r_{1} \operatorname{sgn}(\dot{x}) \sin \psi\left(1+\frac{r_{1}}{c} \cos \psi\right), \frac{r_{1}^{2} r_{2}^{2} x_{2}^{2}}{c^{4}} \times \\
\left.\quad \sin x_{1} \cos x_{1}\left(c+r_{1}^{2} \cos x_{1}^{2}\right), \frac{r_{1}}{2} g \cos x_{1}\right] .
\end{gathered}
$$

Note that,

$$
\hat{k}_{1}=\frac{m_{1}}{\lambda_{1}}, \hat{k}_{2}=m_{2}, \hat{k}_{3}=m_{3}, \hat{k}_{4}=\frac{m_{2}}{\lambda_{2}}, \hat{k}_{5}=m_{1}+m_{2},
$$

and,

$$
\begin{aligned}
& k_{1}=\hat{k}_{1} r_{1}^{2}, k_{2}=\hat{k}_{2} \frac{5 r_{2}^{2} r_{1}^{2}}{4 c^{2}}, k_{3}=\hat{k}_{3} \frac{r_{1}^{4}}{c^{2}}, k_{4}=\left(\hat{k}_{2}+\hat{k}_{3}\right) r_{1}^{2}, \\
& k_{5}=\left(\hat{k}_{2}+2 \hat{k}_{3}\right) \frac{r_{1}^{3}}{c}, k_{6}=-\hat{k}_{4} \frac{r_{2}^{2}}{c^{4}}, k_{7}=\left(\hat{k}_{2}+\hat{k}_{3}\right) \frac{r_{1}}{2 c}, k_{8}=\hat{k}_{2} \frac{r_{2}^{2}}{4 c^{2}}, \\
& k_{9}=\hat{k}_{3} \frac{r_{1}^{2}}{2 c}, k_{10}=g \frac{r_{1}}{2} \hat{k}_{5}, k_{11}=\hat{k}_{3} \mu_{v} g r_{1} .
\end{aligned}
$$

The discrete-time dynamics of the system is required to be able to perform a discrete-time design procedure. Forward Euler approximation to derivatives is used to obtain discretetime dynamics of the system as given below,

$$
\begin{aligned}
& x_{1}^{+}=x_{1}+T x_{2} \\
& x_{2}^{+}=x_{2}+T \frac{\hat{\Phi}_{2 B C}\left(x_{1}, x_{2}\right) \theta}{\hat{\Phi}_{2 A}\left(x_{1}, x_{2}\right) \theta}+T v .
\end{aligned}
$$

The following notation,

$x_{1}=\tilde{x}_{1}, x_{2}=\frac{\tilde{x}_{2}-\tilde{x}_{1}}{T}$,

yields

with 


$$
\begin{aligned}
& \tilde{x}_{1}^{+}=\tilde{x}_{2} \\
& \tilde{x}_{2}^{+}=T^{2} \frac{\hat{\Phi}_{2 B C}\left(\tilde{x}_{1}, \tilde{x}_{2}\right) \theta}{\hat{\Phi}_{2 A}\left(\tilde{x}_{1}, \tilde{x}_{2}\right) \theta}+2 \tilde{x}_{2}-\tilde{x}_{1}+T^{2} v
\end{aligned}
$$

and when $u=2 \tilde{x}_{2}-\tilde{x}_{1}+T^{2} v, \quad \Phi_{2 B C}=T^{2} \hat{\Phi}_{2 B C} \quad$ and $\Phi_{2 A}=\hat{\Phi}_{2 A}$ are considered, the expression for the discretetime dynamics are obtained in the following form,

$\tilde{x}_{1}^{+}=\tilde{x}_{2}$

$\tilde{x}_{2}^{+}=\frac{\Phi_{2 B C}\left(\tilde{x}_{1}, \tilde{x}_{2}\right) \theta}{\Phi_{2 A}\left(\tilde{x}_{1}, \tilde{x}_{2}\right) \theta}+u$.

In the sequel, an indirect adaptive controller construction is derived using immersion and invariance control approach. For the estimation of the parameters, following estimation error is considered.

$z=\hat{\theta}-\theta+\gamma \Phi_{2 A}\left(\tilde{x}_{1}^{-}, \tilde{x}_{2}^{-}\right) \theta \beta\left(\tilde{x}_{1}^{-}\right) \tilde{x}$,

where $0<\gamma<1$ and is such that $\bar{\sigma}\left(\gamma \beta\left(\tilde{x}_{1}^{-}\right) x \Phi_{2 A}\left(\tilde{x}_{1}^{-}, \tilde{x}_{2}^{-}\right)\right)<1$, namely $\left(I-\gamma \beta\left(\tilde{x}_{1}^{-}\right) x \Phi_{2 A}\left(\tilde{x}_{1}^{-}, \tilde{x}_{2}^{-}\right)\right)^{-1}$ exists. Note that,

$\theta=\left(I-\gamma \beta\left(\tilde{x}_{1}^{-}\right) \tilde{x} \Phi_{2 A}\left(\tilde{x}_{1}^{-}, \tilde{x}_{2}^{-}\right)\right)^{-1}(\hat{\theta}-z)$,

and for $\Gamma=\left(I-\gamma \beta\left(\tilde{x}_{1}^{-}\right) x \Phi_{2 A}\left(\tilde{x}_{1}^{-}, \tilde{x}_{2}^{-}\right)\right)^{-1}$, it is rewritten as $\theta=\Gamma(\hat{\theta}-z)$

hence parameter estimations are obtained as

$\theta_{\text {est }}=\Gamma \hat{\theta}$.

Also note that,

$z^{+}=\hat{\theta}^{+}-\theta+\gamma \Phi_{2 A}\left(\tilde{x}_{1}, \tilde{x}_{2}\right) \theta \beta\left(\tilde{x}_{1}\right) \tilde{x}^{+}$.

Therefore,

$$
\begin{aligned}
z^{+}-\alpha & =\hat{\theta}^{+}+(1-\alpha) \theta+\gamma \Phi_{2 A}\left(\tilde{x}_{1}, \tilde{x}_{2}\right) \theta \beta\left(\tilde{x}_{1}\right) \tilde{x}^{+}-\alpha \hat{\theta} \\
- & \alpha \gamma \Phi_{2 A}\left(\tilde{x}_{1}^{-}, \tilde{x}_{2}^{-}\right) \theta \beta\left(\tilde{x}_{1}^{-}\right) \tilde{x} \\
= & \left.\hat{\theta}^{+}+(1-\alpha) \theta+\gamma \Phi_{2 A}\left(\tilde{x}_{1}, \tilde{x}_{2}\right) \theta \beta\left(\tilde{x}_{1}\right)\right)\left[\begin{array}{c}
\tilde{x}_{1}^{+} \\
\tilde{x}_{2}^{+}
\end{array}\right]-\alpha \hat{\theta} \\
& -\alpha \gamma \Phi_{2 A}\left(\tilde{x}_{1}^{-}, \tilde{x}_{2}^{-}\right) \theta \beta\left(\tilde{x}_{1}^{-}\right) \tilde{x} \\
= & \hat{\theta}^{+}-\alpha \hat{\theta}+\gamma \Phi_{2 A}\left(\tilde{x}_{1}, \tilde{x}_{2}\right) \theta \beta\left(\tilde{x}_{1}\right)\left[\frac{\Phi_{2 B C}\left(\tilde{x}_{1}, \tilde{x}_{2}\right) \theta}{\Phi_{2 A}\left(\tilde{x}_{1}, \tilde{x}_{2}\right) \theta}+u\right] \\
- & \alpha \gamma \Phi_{2 A}\left(\tilde{x}_{1}^{-}, \tilde{x}_{2}^{-}\right) \theta \beta\left(\tilde{x}_{1}^{-}\right)\left[\begin{array}{l}
\tilde{x}_{1} \\
\tilde{x}_{2}
\end{array}\right]+(1-\alpha) \theta \\
= & \hat{\theta}^{+}-\alpha \hat{\theta}+\gamma \beta\left(\tilde{x}_{1}\right)\left[\begin{array}{c}
\Phi_{2 B C}\left(\tilde{x}_{1}, \tilde{x}_{2}\right) \theta+\Phi_{2 A}\left(\tilde{x}_{1}, \tilde{x}_{2}\right) \theta u \\
\left.\tilde{x}_{1}, \tilde{x}_{2}\right) \theta \tilde{x}_{2}
\end{array}\right] \\
- & \alpha \gamma \Phi_{2 A}\left(\tilde{x}_{1}^{-}, \tilde{x}_{2}^{-}\right) \theta \beta\left(\tilde{x}_{1}^{-}\right)\left[\begin{array}{l}
\tilde{x}_{1} \\
\tilde{x}_{2}
\end{array}\right]+(1-\alpha) \theta
\end{aligned}
$$

$$
\begin{aligned}
& =\hat{\theta}^{+}-\alpha \hat{\theta}+\gamma \beta\left(\tilde{x}_{1}\right)\left[\begin{array}{l}
\tilde{x}_{2} \Phi_{2 A}\left(\tilde{x}_{1}, \tilde{x}_{2}\right) \Gamma(\hat{\theta}-z), \\
\Phi_{2 B C}\left(\tilde{x}_{1}, \tilde{x}_{2}\right) \Gamma(\hat{\theta}-z) \\
+u \Phi_{2 A}\left(\tilde{x}_{1}, \tilde{x}_{2}\right) \Gamma(\hat{\theta}-z)
\end{array}\right] \\
& -\alpha \gamma \Phi_{2 A}\left(\tilde{x}_{1}^{-}, \tilde{x}_{2}^{-}\right) \Gamma(\hat{\theta}-z) \beta\left(\tilde{x}_{1}^{-}\right)\left[\begin{array}{l}
\tilde{x}_{1} \\
\tilde{x}_{2}
\end{array}\right]+(1-\alpha) \Gamma(\hat{\theta}-z),
\end{aligned}
$$

is obtained. Let's select the update law as

$$
\begin{aligned}
& \hat{\theta}^{+}=\alpha \hat{\theta}-\gamma \beta\left(\tilde{x}_{1}\right)\left[\begin{array}{c}
\tilde{x}_{2} \Phi_{2 A}\left(\tilde{x}_{1}, \tilde{x}_{2}\right) \Gamma \hat{\theta} \\
\Phi_{2 B C}\left(\tilde{x}_{1}, \tilde{x}_{2}\right) \Gamma \hat{\theta}+u \Phi_{2 A}\left(\tilde{x}_{1}, \tilde{x}_{2}\right) \Gamma \hat{\theta}
\end{array}\right] \\
& +\alpha \gamma \Phi_{2 A}\left(\tilde{x}_{1}^{-}, \tilde{x}_{2}^{-}\right) \Gamma \hat{\theta} \beta\left(\tilde{x}_{1}^{-}\right)\left[\begin{array}{l}
\tilde{x}_{1} \\
\tilde{x}_{2}
\end{array}\right]+(\alpha-1) \Gamma \hat{\theta} .
\end{aligned}
$$

Then the estimation error dynamics becomes

$$
\begin{aligned}
z^{+}= & \alpha z-\gamma \beta\left(\tilde{x}_{1}\right)\left[\begin{array}{c}
\tilde{x}_{2} \Phi_{2 A}\left(\tilde{x}_{1}, \tilde{x}_{2}\right) \Gamma \\
\Phi_{2 B C}\left(\tilde{x}_{1}, \tilde{x}_{2}\right) \Gamma+u \Phi_{2 A}\left(\tilde{x}_{1}, \tilde{x}_{2}\right) \Gamma
\end{array}\right] z \\
& +\alpha \gamma \beta\left(\tilde{x}_{1}^{-}\right)\left[\begin{array}{c}
\tilde{x}_{1} \\
\tilde{x}_{2}
\end{array}\right] \Phi_{2 A}\left(\tilde{x}_{1}^{-}, \tilde{x}_{2}^{-}\right) \Gamma z+(\alpha-1) \Gamma z .
\end{aligned}
$$

Using the following notation

$$
\begin{aligned}
& \Phi=\left[\begin{array}{c}
\tilde{x}_{2} \Phi_{2 A}\left(\tilde{x}_{1}, \tilde{x}_{2}\right) \\
\Phi_{2 B C}\left(\tilde{x}_{1}, \tilde{x}_{2}\right)+u \Phi_{2 A}\left(\tilde{x}_{1}, \tilde{x}_{2}\right)
\end{array}\right], \\
& \Pi=\left[\begin{array}{c}
\tilde{x}_{1} \\
\tilde{x}_{2}
\end{array}\right] \Phi_{2 A}\left(\tilde{x}_{1}^{-}, \tilde{x}_{2}^{-}\right),
\end{aligned}
$$

(34) can be rewritten as,

$$
z^{+}=\left(\alpha I-\gamma \beta\left(\tilde{x}_{1}\right) \Phi \Gamma+\alpha \gamma \beta\left(\tilde{x}_{1}^{-}\right) \Pi \Gamma+(\alpha-1) \Gamma\right) z
$$

Let's select,

$$
\beta=\beta\left(x_{1}\right)=\left(I+M^{+T} M^{+}+K_{1}^{+}+K_{2}^{+}\right)^{-1} M^{+T}
$$

and

$$
\begin{array}{ll}
0<\alpha<1-\frac{0.5 \kappa_{1}}{1+\bar{\sigma}(\Gamma)}, & 0<\gamma<\frac{0.5 \kappa_{2}}{1+\alpha}, \\
0<\kappa_{1}, \kappa_{2}<1, & \kappa_{1}>\kappa_{2},
\end{array}
$$

where

$$
\begin{aligned}
& M=\gamma x \Phi_{2 A}\left(\tilde{x}_{1}^{-}, \tilde{x}_{2}^{-}\right), \\
& K_{1}^{+}=M^{T} M+\bar{\sigma}\left(K_{1}\right) I_{p}+K_{2}, K_{1}(0) \geq 0, \\
& K_{2}=\bar{\sigma}\left(M^{T} \Pi\right) I_{p}+\bar{\sigma}\left(M^{+T} \Phi\right) I_{p} .
\end{aligned}
$$

Note that,

$\beta=\beta\left(\tilde{x}_{1}\right)=\left(R^{+}\right)^{-1} M^{+T}$ and $\beta^{-}=\beta\left(\tilde{x}_{1}^{-}\right)=R^{-1} M^{T}$ where

$$
\begin{aligned}
& R=I+M^{T} M+K_{1}+K_{2}, \\
& L=I+K_{1}+K_{2},
\end{aligned}
$$


hence,

$$
\begin{aligned}
z^{+}= & \left(\alpha I-\gamma\left(R^{+}\right)^{-1} M^{+T} \Phi L^{-1} R+\right. \\
& \left.\alpha \gamma R^{-1} M^{T} \Pi L^{-1} R+(\alpha-1) \Gamma\right) z .
\end{aligned}
$$

Proposition: Consider the system (43) in which $\alpha$ and $\gamma$ satisfy (37). The zero equilibrium of the system is asymptotically stable, namely $\lim _{k \rightarrow \infty} z(k)=0$.

Proof: Consider the candidate Lyapunov function $V=z^{T} z$ and,

$$
\begin{aligned}
\Delta V & =z^{T+} z^{+}-z^{T} z \\
& =z^{T}\left[\Lambda^{T} \Lambda-I\right] z
\end{aligned}
$$

where

$$
\begin{aligned}
\Lambda= & \alpha I-\gamma\left(R^{+}\right)^{-1} M^{+T} \Phi L^{-1} R+\alpha \gamma R^{-1} M^{T} \Pi L^{-1} R \\
& +(\alpha-1) \Gamma .
\end{aligned}
$$

In (44), $\bar{\sigma}\left(\left(R^{+}\right)^{-1} M^{+T} \Phi L^{-1} R\right) \leq 1, \bar{\sigma}\left(R^{-1} M^{T} \Pi L^{-1} R\right) \leq 1$. Let $\tilde{I} \triangleq\left(R^{+}\right)^{-1} M^{+T} \Phi L^{-1} R, \hat{I} \triangleq R^{-1} M^{T} \Pi L^{-1} R$ and rewrite (43) as

$$
\Lambda=\alpha(I+\Gamma)-\Gamma \pm \gamma(\tilde{I} \pm \alpha \hat{I})
$$

consider $\alpha$ and $\gamma$ satisfy (37), then

$$
\Lambda=I-(I+\Gamma) \frac{0.5 \kappa_{1}+\xi_{1}}{1+\bar{\sigma}(\Gamma)} \pm \frac{0.5 \kappa_{2}-\xi_{2}}{1+\alpha}(\tilde{I} \pm \alpha \hat{I})
$$

where $\xi_{1}, \xi_{1}>0$ are such that $\alpha, \gamma>0$. Thus $\bar{\sigma}(\Lambda)<1$ and this makes $\Delta V<0$. Therefore $\lim _{k \rightarrow \infty} z(k)=0$.

Remark: In the estimation of the parameters the "look-ahead" values of the state variable $x_{i}$ is required. These values are calculated utilizing the system equations (26) with parameter values obtained in the previous estimations.

The back-stepping construction of the controller is performed in the sequel.

Consider system equations (26) and denote $\hat{x}_{1}=\tilde{x}_{1}-\tilde{x}_{1}^{*}$, then,

$\hat{x}_{1}^{+}=\tilde{x}_{2}-\tilde{x}_{1}^{*+}$

and assume $\tilde{x}_{2}$ as virtual input, note that $\hat{x}_{2}=\tilde{x}_{2}-\tilde{x}_{2}^{*}$ and select $\tilde{x}_{2}^{*}=\tilde{x}_{1}^{*+}$, thus

$$
\hat{x}_{1}^{+}=\hat{x}_{2}
$$

and note that

$$
\hat{x}_{2}^{+}=\frac{\Phi_{2 B C}\left(\tilde{x}_{1}, \tilde{x}_{2}\right) \theta}{\Phi_{2 A}\left(\tilde{x}_{1}, \tilde{x}_{2}\right) \theta}-\tilde{x}_{1}^{*++}+u
$$

Taking the control signal $u$ as

$$
u=-\frac{\Phi_{2 B C}\left(\tilde{x}_{1}, \tilde{x}_{2}\right) \theta_{e s t}}{\Phi_{2 A}\left(\tilde{x}_{1}, \tilde{x}_{2}\right) \theta_{e s t}}+\tilde{x}_{1}^{*++}
$$

yields
$\hat{x}_{2}^{+}=\frac{\Phi_{2 B C}\left(\tilde{x}_{1}, \tilde{x}_{2}\right) \theta}{\Phi_{2 A}\left(\tilde{x}_{1}, \tilde{x}_{2}\right) \theta}-\frac{\Phi_{2 B C}\left(\tilde{x}_{1}, \tilde{x}_{2}\right) \theta_{e s t}}{\Phi_{2 A}\left(\tilde{x}_{1}, \tilde{x}_{2}\right) \theta_{e s t}}$.

This can be rewritten as,

$$
\hat{x}_{2}^{+}=\frac{\Phi_{2 B C}\left(\tilde{x}_{1}, \tilde{x}_{2}\right) \theta \Phi_{2 A}\left(\tilde{x}_{1}, \tilde{x}_{2}\right) \theta_{e s t}-\Phi_{2 B C}\left(\tilde{x}_{1}, \tilde{x}_{2}\right) \theta_{\text {est }} \Phi_{2 A}\left(\tilde{x}_{1}, \tilde{x}_{2}\right) \theta}{\Phi_{2 A}\left(\tilde{x}_{1}, \tilde{x}_{2}\right) \theta \Phi_{2 A}\left(\tilde{x}_{1}, \tilde{x}_{2}\right) \theta_{\text {est }}},
$$$$
\hat{x}_{2}^{+}=\frac{\Phi_{2 B C}\left(\tilde{x}_{1}, \tilde{x}_{2}\right)\left(\theta_{e s t}-\Gamma z\right) \Phi_{2 A}\left(\tilde{x}_{1}, \tilde{x}_{2}\right) \theta_{e s t}}{\Phi_{2 A}\left(\tilde{x}_{1}, \tilde{x}_{2}\right) \theta \Phi_{2 A}\left(\tilde{x}_{1}, \tilde{x}_{2}\right) \theta_{\text {est }}}
$$

$$
-\frac{\Phi_{2 B C}\left(\tilde{x}_{1}, \tilde{x}_{2}\right) \theta_{e s t} \Phi_{2 A}\left(\tilde{x}_{1}, \tilde{x}_{2}\right)\left(\theta_{\text {est }}-\Gamma z\right)}{\Phi_{2 A}\left(\tilde{x}_{1}, \tilde{x}_{2}\right) \theta \Phi_{2 A}\left(\tilde{x}_{1}, \tilde{x}_{2}\right) \theta_{\text {est }}},
$$

$\hat{x}_{2}^{+}=\frac{\Phi_{2 B C}\left(\tilde{x}_{1}, \tilde{x}_{2}\right) \theta_{e s t} \Phi_{2 A}\left(\tilde{x}_{1}, \tilde{x}_{2}\right) \Gamma z-\Phi_{2 B C}\left(\tilde{x}_{1}, \tilde{x}_{2}\right) \Gamma z \Phi_{2 A}\left(\tilde{x}_{1}, \tilde{x}_{2}\right) \theta_{e s t}}{\Phi_{2 A}\left(\tilde{x}_{1}, \tilde{x}_{2}\right) \theta \Phi_{2 A}\left(\tilde{x}_{1}, \tilde{x}_{2}\right) \theta_{e s t}}$,

hence, the closed loop system dynamics are obtained as,

$\hat{x}_{1}^{+}=\hat{x}_{2}$

$\hat{x}_{2}^{+}=\frac{\Phi_{2 B C}\left(\tilde{x}_{1}, \tilde{x}_{2}\right) \theta_{e s t} \Phi_{2 A}\left(\tilde{x}_{1}, \tilde{x}_{2}\right) \Gamma z-\Phi_{2 A}\left(\tilde{x}_{1}, \tilde{x}_{2}\right) \theta_{e s t} \Phi_{2 B C}\left(\tilde{x}_{1}, \tilde{x}_{2}\right) \Gamma z}{\Phi_{2 A}\left(\tilde{x}_{1}, \tilde{x}_{2}\right) \theta \Phi_{2 A}\left(\tilde{x}_{1}, \tilde{x}_{2}\right) \theta_{\text {est }}}$.

Note that, when $z=0$, these dynamics become,

$\hat{x}_{1}^{+}=\hat{x}_{2}$

$\hat{x}_{2}^{+}=0$.

Thus, considering the Proposition, it can be claimed that if the update law (33) together with (36) is used, the control objective is achieved. Note that,

$\tau=A\left(\theta_{\text {est }}\right) \tilde{\tau}=\left.A(\theta+z) \tilde{\tau}\right|_{z=0}=A(\theta) \tilde{\tau}$.

\section{SIMULATION RESULTS}

In this paper basically the regulation problem is considered therefore the simulation results for $\tilde{x}_{1}^{*}=0$ are presented in the sequel. In the simulations, system parameters are taken as $g=9.81 \mathrm{kgm}^{2} / \mathrm{s}, \quad r_{1}=0.3 \mathrm{~m}, \quad r_{2}=1 \mathrm{~m}, \quad m_{1}=0.45 \mathrm{~kg}$, $m_{2}=1.5 \mathrm{~kg}, \quad m_{3}=0.5 \mathrm{~kg}, \lambda_{1}=3, \lambda_{2}=3$ which yields $\theta=[0.225,1.5,0.5,0.75,1.95]$ and $\mathrm{T}=0.01$ is used to obtain discrete-time dynamics. Estimator parameters are selected as $\kappa_{1}=0.9, \quad \kappa_{2}=0.5, \quad \gamma=0.1$ and $\alpha=0.3$. The simulations are run with the initial conditions $\tilde{x}=[3,0.5]$ and $\hat{\theta}(0)=[10,10,10,10,10]$. Figure 2 illustrates the time histories of the parameter estimation errors $\mathrm{Z}=\left[z_{1}, z_{2}, z_{3}, z_{4}, z_{5}\right]$ which converge to zero, while Figure 3, Figure 4 and Figure 5 illustrate the time history of the state angular position of first joint $x_{1}$, angular velocity of first joint $x_{2}$ and the edge position $x$ for both the adaptive control case and the known parameter control case (Dasheddot lines corresponds to adaptive control, solid lines corresponds to known parameter control). Note that, the edge position is calculated using the relation $x=r_{1} \cos x_{1}+c$. 


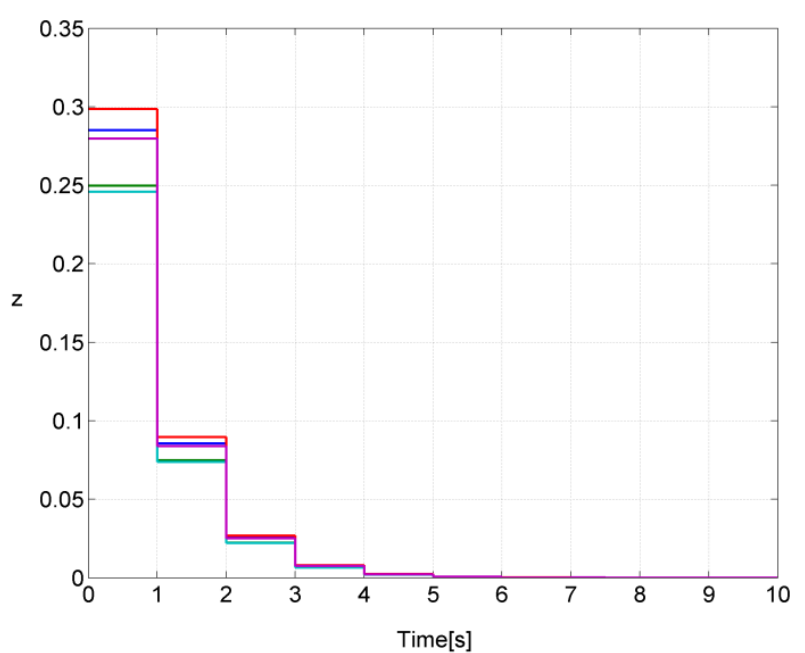

Fig. 2. Time history of the parameter estimation error $z$.

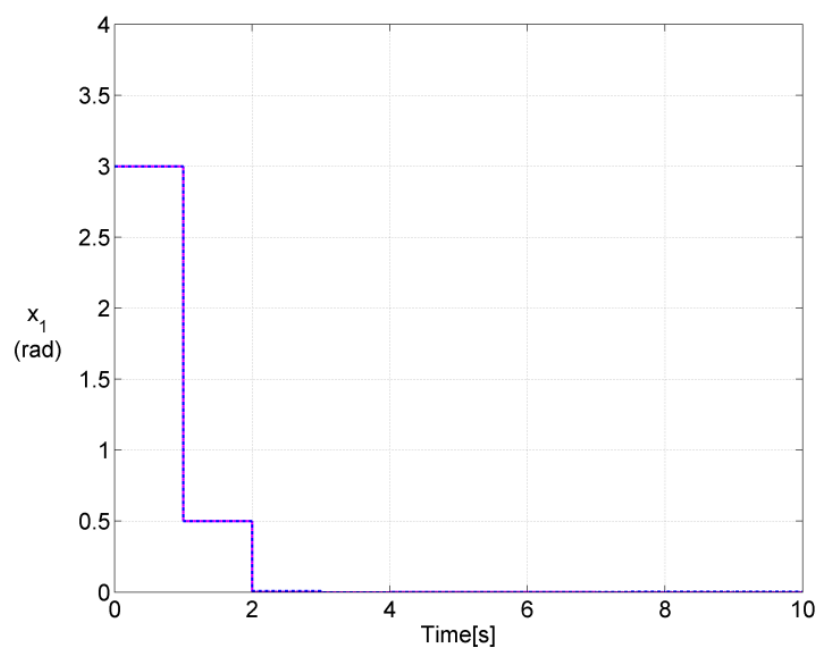

Fig. 3. Time history of the variable $x_{1}$ (angular position)

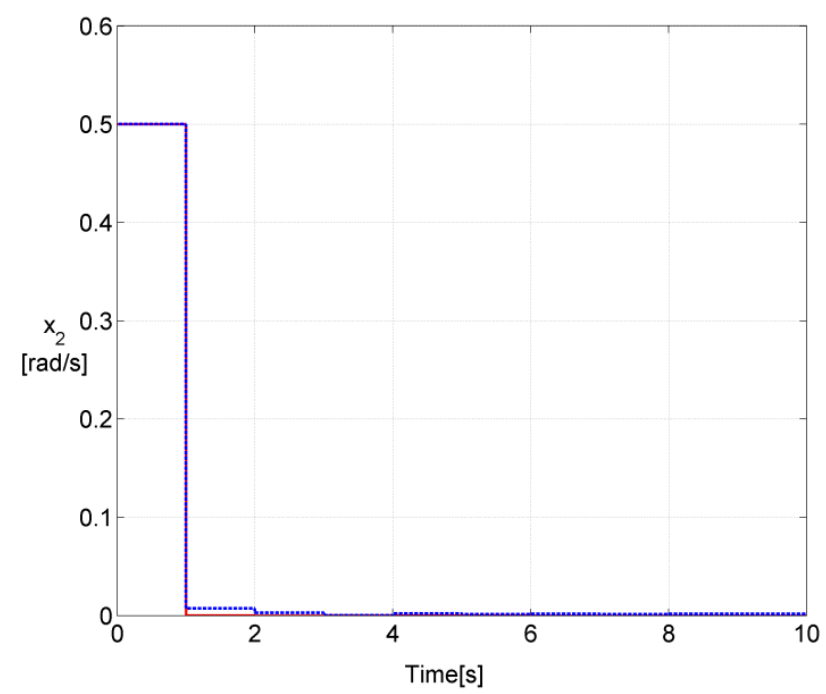

Fig. 4. Time history of the variable $x_{2}$ (angular velocity)

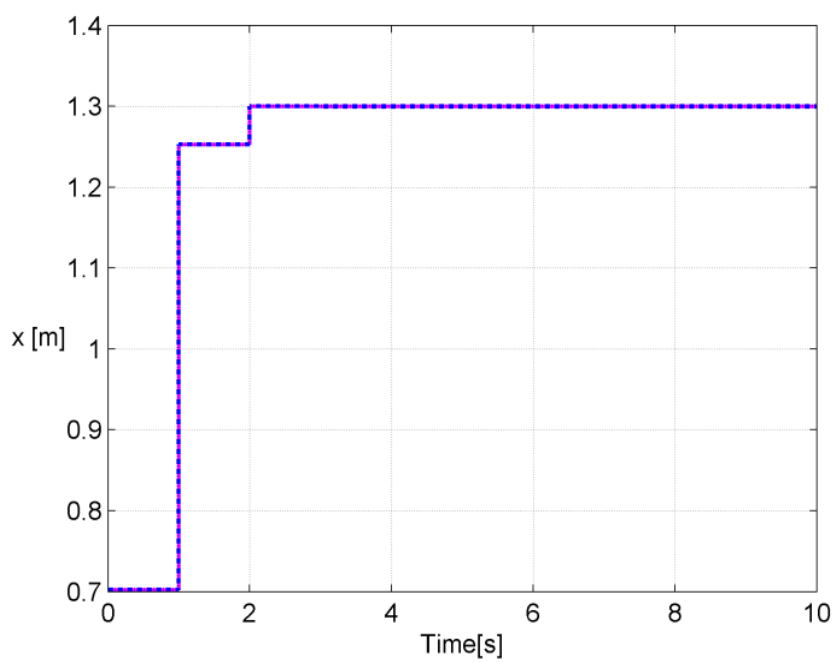

Fig. 5. Time history of the edge position $x$.

\section{REFERENCES}

Astolfi, A., D. Karagiannis, and R. Ortega (2008). Nonlinear and Adaptive Control with Applications. Springer.

Astolfi, A., D. Karagiannis, and R. Ortega (2007). Stabilization of uncertain nonlinear systems via immersion and invariance. European Journal of Control, vol. 13, pp. 204-220.

Fung, R.F. and C.F. Chang (2009). Force/Motion Slidingmode Control Of Three Typical mechanisms. Asian Journal of Control, vol. 11, no. 2, pp. 196-210.

Ha, J., R. Fung, K.Y. Chen, and S.C. Hsien (2006). Dynamic modeling and identification of a slider-crank mechanism. Journal of Sound and Vibration, vol. 289, pp. 1019-1044.

Karagiannis, D. and A. Astolfi (2008). Nonlinear adaptive control of systems in feedback form:an alternative to adaptive backstepping. Systems and Control Letters, vol. 57, pp. 773739.

Kao, C.C., C.W. Chuang, and R.F. Fung (2006). The self-tuning PID control in a slider-crank mechanism system by applying particle swarm optimization approach. Mechatronics, vol. 16, no. 8, pp. 513-522.

Komaita, Y., and K. Furuta (2008). Energy control of slider-crank mechanism. SICE Annual Conference, pp. 2399 - 2403

Lin, F.J., Y.S. Lin, and S.L. Chiu (1998). Slider-crank mechanism control using adaptive computed torque technique. Control Theory and Applications, IEE Proceedings -, vol. 145, pp. 364-376.

Lin, F.J., R.F. Fung, H.H. Lin, and C.M. Hong, (2001). A supervisory fuzzy neural network controller for slider-crank mechanism. Mechatronics, vol. 11, pp. 227-250.

Saitoh, M., and K. Furuta (2007). Generalized minimum variance control of slider-crank mechanism. SICE Annual Conference, pp. $2205-2209$.

Wai, R.J. and F.J. Lin (1998). A fuzzy neural network controller with adaptive learning rates for nonlinear slider-crank mechanism. Neurocomputing, vol. 20, pp.295-320.

Yalcin, Y. and A. Astolfi (2012). Immersion and invariance adaptive control for discrete time systems in strict feedback form. Systems and Control Letters, vol. 61, no. 12, pp. 1132-1137.

Yalcin, Y. and A. Astolfi (2011). Discrete time immersion and invariance adaptive control for systems in strict feedback form. Proceedings of the 50th IEEE Conference on Decision and Control and European Control Conference (CDC-ECC 2011), Orlando, FL, USA, pp. 343-347. 